\title{
THEORETICAL PEARLS \\ Applications of Plotkin-terms: partitions and morphisms for closed terms
}

\author{
RICHARD STATMAN \\ Department of Mathematics, Carnegie-Mellon University, \\ Pittsburgh, Pennsylvania 15213, USA. \\ (e-mail: Rick.Statman@andrew.cmu.edu) \\ HENK BARENDREGT \\ Department of Computer Science, Catholic University, \\ Box 9102, 6500 HC Nijmegen, The Netherlands. \\ (e-mail: henk@cs.kun.nl)
}

\begin{abstract}
This theoretical pearl is about the closed term model of pure untyped lambda-terms modulo $\beta$-convertibility. A consequence of one of the results is that for arbitrary distinct combinators (closed lambda terms) $M, M^{\prime}, N, N^{\prime}$ there is a combinator $H$ such that

$$
H M=H M^{\prime} \neq H N=H N^{\prime} .
$$

The general result, which comes from Statman [1998], is that uniformly r.e. partitions of the combinators, such that each "block" is closed under $\beta$-conversion, are of the form $\left\{H^{-1}\{M\}\right\}_{M \in \Lambda}$. This is proved by making use of the idea behind the so-called Plotkinterms, originally devised to exhibit some global but non-uniform applicative behavior. For expository reasons we present the proof below. The following consequences are derived: a characterization of morphisms and a counter-example to the perpendicular lines lemma for $\beta$-conversion.
\end{abstract}

\section{Introduction}

We use notations from recursion theory and lambda calculus, see Rogers [1987] and Barendregt [1984].

Notation. (i) $\varphi_{e}$ is the $e$-th partial recursive function of one argument.

(ii) $W_{e}=\operatorname{dom}\left(\varphi_{e}\right) \subseteq \mathbb{N}$ is the r.e. set with index $e$.

(iii) $\Lambda$ is the set of lambda-terms and $\Lambda^{\varnothing}$ is the set of closed-lambda terms (combinators).

(iv) $\mathcal{W}_{e}=\left\{M \in \Lambda^{\emptyset} \mid \# M \in W_{e}\right\} \subseteq \Lambda^{\emptyset}$; here $\# M$ is the code of the term $M$.

1.1. Definition. (i) Inspired by Visser [1980] we define a Visser-partition (Vpartition) of $\Lambda^{\emptyset}$ to be a family $\left\{\mathcal{W}_{e}\right\}_{e \in S}$ such that 
(1) $S \subseteq \mathbb{N}$ is an r.e. set

(2) $\forall e \in S \forall M, N\left(M \in \mathcal{W}_{e} \& N=M\right) \Rightarrow N \in \mathcal{W}_{e}$.

(3) $\mathcal{W}_{e} \cap \mathcal{W}_{e^{\prime}} \neq \emptyset \Rightarrow \mathcal{W}_{e}=\mathcal{W}_{e^{\prime}}$

(ii) A family $\left\{\mathcal{W}_{e}\right\}_{e \in S}$ is a pseudo-V-partition if it satisfies just 1 and 2 .

1.2. Definition. Let $\left\{\mathcal{W}_{e}\right\}_{e \in S}$ be a V-partition.

1. The partition is said to be covering if $\bigcup_{e \in S} \mathcal{W}_{e}=\Lambda^{\varnothing}$.

2. The partition is said to be inhabited if $\forall e \in S \mathcal{W}_{e} \neq \emptyset$.

3. A V-partition $\left\{\mathcal{W}_{e}\right\}_{e \in S^{\prime}}$ is said to be (extensionally) equivalent with $\left\{\mathcal{W}_{e}\right\}$ if these families define the same collection of non-empty sets, i.e. if

$$
\left\{\mathcal{W}_{e} \mid e \in S \& \mathcal{W}_{e} \neq \emptyset\right\}=\left\{\mathcal{W}_{e} \mid e \in S^{\prime} \& \mathcal{W}_{e} \neq \emptyset\right\}
$$

1.3. ExAmple. Let $H$ be some given combinator. Define

$$
\mathcal{W}_{e(M, H)}=\left\{N \in \Lambda^{\varnothing} \mid H N=H M\right\},
$$

Then $\left\{\mathcal{W}_{e}\right\}_{e \in S_{H}}$, with $S_{H}=\left\{e(M, H) \mid M \in \Lambda^{\varnothing}\right\}$, is an example of a covering and inhabited V-partition. We denote this V-partition by $\left\{\mathcal{W}_{e(M, H)}\right\}_{M \in \Lambda} \phi$.

1.4. Proposition. (i) Every $V$-partition is effectively equivalent to an inhabited one.

(ii) Every V-partition can effectively be extended to a covering one.

Proof. (i) Given $\left\{\mathcal{W}_{e}\right\}_{e \in S}$ define $S^{\prime}=\left\{e \in S \mid \mathcal{W}_{e} \neq \emptyset\right\}$. Then $\left\{\mathcal{W}_{e}\right\}_{e \in S^{\prime}}$ is the required modified partition.

(ii) Given $\left\{\mathcal{W}_{e}\right\}_{e \in S}$ define

$$
\mathcal{W}_{e(M)}=\left\{N \mid N=M \vee \exists e \in S M, N \in \mathcal{W}_{e}\right\}
$$

Then $\left\{\mathcal{W}_{e(M)}\right\}_{M \in \Lambda} \emptyset$ is the required V-partition.

The main theorem comes in two version. The second more sharp version is needed for the construction of so called inevitably consistent equations, see Statman [1999].

1.5. Theorem (Main theorem). (i) Let $\left\{\mathcal{W}_{e}\right\}_{e \in S}$ be a V-partition. Then one can construct effectively a combinator $H$ such that for all $M, N \in \Lambda^{\emptyset}$

$$
H M=H N \Leftrightarrow M=N \vee \exists e \in S M, N \in \mathcal{W}_{e} .
$$

The construction of $H$ is effective in the code of the underlying r.e. set $S$.

(ii) Let $\left\{\mathcal{W}_{e}\right\}_{e \in S}$ be a pseudo-V-partition. Then one can construct effectively a combinator $H$ such that if $\left\{\mathcal{W}_{e}\right\}_{e \in S}$ is an actual $V$-partition, then $(*)$ holds.

The theorem will be proved in $\S 2$. It has several consequences. In order to state these we have to formulate the notion of morphism on $\Lambda^{\emptyset}$ and the so-called perpendicular lines lemma.

1.6. Definition. Let $\varphi: \Lambda^{\varnothing} \rightarrow \Lambda^{\varnothing}$ be a map. Then $\varphi$ is a morphism if

1. $\varphi(M)=\mathrm{Ec}_{f(\# M)}$, for some recursive function $f$.

2. $M=N \Rightarrow \varphi(M)=\varphi(N)$. 
1.7. LemMA. (i) Let $F$ be a combinator and define $\varphi_{H}(M) \equiv H M$. Then $\varphi_{H}$ is a morphism.

(ii) Let $F, G$ be combinators such that for all $M \in \Lambda^{\varnothing}$ there exists a unique $N \in \Lambda^{\varnothing}$ with $F M=G N$. Then there is a map $\varphi_{F, G}$ such that $F M=G \varphi_{F, G}(M)$, for all $M$, which is a morphism.

Proof. (i) For the coding \# let app be the recursive function such that \# $(P Q)$ $=\operatorname{app}(\# P, \# Q)$. Define $f(m)=\operatorname{app}(\# H, m)$. Then $\varphi_{H}(M)=\operatorname{Ec}_{f(\# M)}$. It is obvious that $\varphi_{H}$ preserves $\beta$-equality.

(ii) Let $R(m, n)$ be an r.e. relation. The we have $R(m, n) \Leftrightarrow \exists z T(m, n, z)$, for some recursive $T$. Let $\langle n, z\rangle$ be a recursive pairing with recursive inverses $<n, z>.0=n,\langle n, z>.1=z$. Define ( $\mu$ is the least number operator)

$$
\iota_{n} \cdot R(m, n)=(\mu p . T(m, p .0, p .1)) \cdot 0 .
$$

Then $\exists n \in \mathbb{N} R(m, n) \Rightarrow R\left(m, \iota_{n} \cdot R(m, n)\right)$. In order to construct the morphism $\varphi_{F, G}$, define

$$
f(m)=\iota_{n} \cdot F\left(E \mathbf{c}_{m}\right)=G\left(E \mathbf{c}_{n}\right) .
$$

By the assumption (existence) $f$ is total. Define $\varphi_{F, G}(M)=\operatorname{Ec}_{f(\# M)}$. Now $f(\# M)=$ $n \Rightarrow F\left(E \mathbf{c}_{n}\right)=G\left(E \mathbf{c}_{n}\right)$. Therefore $F M=G \varphi_{F, G}(M)$, for all $M$. The condition

$$
M=M^{\prime} \Rightarrow \varphi_{F, G}(M)=\varphi_{F, G}\left(M^{\prime}\right)
$$

holds by the assumption (unicity).

One may wonder whether dropping the unicity condition in lemma 1.7 (ii) one may obtain a morphism by making a right uniformization. This is not the case.

1.8. Proposition. There exists combinators $F, G$ such that $\forall M \exists N F M=G N$ but without any morphism satisfying $\forall M F M=G \varphi(N)$.

Proof. Let $\Delta=\mathrm{Y} \Omega$ and define $F=\lambda x .\langle x, \Delta, \mathrm{I}\rangle$ and $G=\lambda y .\langle\mathrm{E} y, y \Omega \Delta, y \mathrm{I}\rangle$. Then, see Statman [1986],

$$
F M={ }_{\beta} G N \Leftrightarrow\left(N={ }_{\beta} c_{n} \vee N={ }_{\beta} \mathrm{I}\right) \& \mathrm{E} N={ }_{\beta} M .
$$

Any morphism $\varphi$ such that $F M=G \varphi(M)$ would solve the convertibility problem recursively: one has by (1)

$$
M=M^{\prime} \Leftrightarrow \varphi(M)=\varphi\left(M^{\prime}\right),
$$

and since $\varphi(M), \varphi\left(M^{\prime}\right)$ have nf's by (1), the RHS of (2) is decidable.

1.9. Proposition. Not every morphism is of the form $\varphi_{H}$.

Proof. Let $F, G \in \Lambda^{\emptyset}$ be such that $F \circ G=\mathrm{I}$. Then $F, G$ determine a so-called inner model $\llbracket \llbracket \llbracket \rrbracket^{F, G}$ as follows.

$$
\begin{aligned}
\llbracket x \rrbracket & =x ; \\
\llbracket P Q \rrbracket & =F \llbracket P \rrbracket \llbracket Q \rrbracket ; \\
\llbracket \lambda x . P \rrbracket & =G(\lambda x . \llbracket P \rrbracket) .
\end{aligned}
$$


Using the condition on $F, G$ it can be proved that

$$
M={ }_{\beta} N \Rightarrow \llbracket M \rrbracket=\llbracket N \rrbracket .
$$

Therefore defining $\varphi(M)=\llbracket M \rrbracket$ we obtain a morphism.

Now take $F \equiv \lambda y . u \mathrm{I}, \Gamma \equiv \lambda x y . y x$. Then indeed $F \circ G=\mathrm{I}$ and for the resulting inner model one has $\llbracket I \rrbracket=\lambda y . y \mid$ and $\llbracket \Omega \rrbracket=(\lambda y . y(\lambda z . z \mid z)) \mathrm{I}(\lambda y . y(\lambda z . z \mid z))$.

Suppose towards a contradiction that the resulting $\varphi$ is of the form $\varphi_{H}$. Then $H \mathrm{I}=\lambda y . y \mathrm{I}$, so $H$ is solvable and hence has a $\operatorname{hnf} \lambda x_{1} \ldots x_{n} \cdot \cdot_{i} M_{1} \ldots M_{m}$. But $H \Omega=$ $(\lambda y . y(\lambda z . z \mid z)) \mathbf{I}(\lambda y . y(\lambda z . z \mid z))$, which is unsolvable. Therefore the head-variable $x_{i}$ is $x_{1}$. But then $H \Omega=\lambda x_{2} \ldots x_{n} . \Omega M_{1}^{*} \ldots M_{m}^{*}$ which is not of the correct form.

The following is a corollary to the main theorem.

1.10. Corollary. Every morphism $\varphi$ is of the form $\varphi_{F, G}$.

Proof. Let $\varphi$ be a given morphism. Define

$$
\mathcal{W}_{e(N)}=\left\{Z \mid \exists M \in \Lambda^{\varnothing}\left[\varphi(M)=N \&\left[Z=\left\langle\mathbf{c}_{0}, M\right\rangle \vee Z=\left\langle\mathbf{c}_{1}, N\right\rangle\right]\right]\right\} .
$$

Then $\left\{\mathcal{W}_{e(N)}\right\}$ is a V-partition. By the main theorem there exists an $H$ such that

$$
\begin{aligned}
H\left\langle\mathbf{c}_{0}, M\right\rangle=H\left\langle\mathbf{c}_{1}, N\right\rangle & \Leftrightarrow\left\langle\mathbf{c}_{0}, M\right\rangle=\left\langle\mathbf{c}_{1}, N\right\rangle \vee N=\varphi(M) \\
& \Leftrightarrow \quad N=\varphi(M) .
\end{aligned}
$$

Define

$$
\begin{aligned}
& F=\lambda m \cdot H\left\langle\mathbf{c}_{0}, m\right\rangle ; \\
& G=\lambda n \cdot H\left\langle\mathbf{c}_{1}, n\right\rangle .
\end{aligned}
$$

Then $F M=G N \Leftrightarrow N=\varphi(M)$. Therefore $\varphi=\varphi_{F, G}$.

Note that for a given morphism $\varphi$ one can define by

$$
\mathcal{W}_{e(M, \varphi)}=\left\{N \in \Lambda^{\varnothing} \mid \varphi(M)=\varphi(N)\right\} .
$$

This is an inhabited V-partition. It is not difficult to show that that each V-partition is equivalent to one of the form $\left\{\mathcal{W}_{e(M, \varphi)}\right\}$. Note that $\left\{\mathcal{W}_{e(M, H)}\right\}=\left\{\mathcal{W}_{e\left(M, \varphi_{H}\right)}\right\}$, see lemma 1.7. The following result shows that covering $\mathrm{V}$-partitions are always of this more restricted form.

1.11. Corollary. If $\left\{\mathcal{W}_{e}\right\}$ is a covering V-partition, then $\left\{\mathcal{W}_{e}\right\}$ is equivalent to $\left\{\mathcal{W}_{e(M, H)}\right\}_{M \in \Lambda^{\varnothing}}$ for some $H$, effectively found from $\left\{\mathcal{W}_{e}\right\}$.

Proof. Let $H$ be the combinator constructed effectively from $\left\{\mathcal{W}_{e}\right\}$. We will show that $\mathcal{W}_{e(M, H)}=\{N \mid H N=H M\}$ is equivalent to $\left\{\mathcal{W}_{e}\right\}$. Claim. For $N \in \mathcal{W}_{e}$ one has $\mathcal{W}_{e}=\mathcal{W}_{e(M, H)}$. Indeed,

$$
\begin{aligned}
N \in \mathcal{W}_{e} & \Leftrightarrow M=N \vee M, N \in \mathcal{W}_{e} \\
& \Leftrightarrow H N=H M \\
& \Leftrightarrow N \in \mathcal{W}_{e(M, H)} .
\end{aligned}
$$


Therefore, noting that $M \in \mathcal{W}_{e(M, H)}$,

$$
\left\{\mathcal{W}_{e} \mid M \in \Lambda^{\emptyset}, \mathcal{W}_{e} \neq \emptyset\right\} \subseteq\left\{\mathcal{W}_{e(M, H)} \mid \mathcal{W}_{e(M, H)} \neq \emptyset, M \in \Lambda^{\emptyset}\right\} .
$$

The converse inclusion holds also, since every $M$ belongs to some $\mathcal{W}_{e}$ and hence $\mathcal{W}_{e(M, H)}=\mathcal{W}_{e}$ for this $e$.

The following theorem states that if a combinator, seen as function of $n$ arguments, is constant-modulo Böhm-tree equality-on $n$ perpendicular lines, then it is constant everywhere.

1.12. Theorem (Perpendicular lines lemma). Let $F$ be a combinator. Suppose that for $n \in \mathbb{N}$ there are combinators $M_{i j}, 1 \leq i \neq j \leq n$, and $N_{1}, \ldots, N_{n}$ such that for all combinators $Z$ one has $(\cong$ denotes Böhm-tree equality, i.e. $M \cong N \Leftrightarrow B T(M)=$ $B T(N))$

$$
\begin{array}{ccccccc}
F & Z & M_{12} & \ldots & M_{1 n-1} & M_{1 n} \cong N_{1} ; \\
F & M_{21} & Z & \ldots & M_{2 n-1} & M_{2 n} \cong N_{2} ; \\
& \multicolumn{7}{l}{} \\
F & & \ldots & \\
F & M_{n 1} & M_{n 2} & \ldots & M_{n n-1} & Z & \cong N_{n} .
\end{array}
$$

Then for all $P_{1}, \ldots, P_{n} \in \Lambda^{\varnothing}$ one has

$$
F P_{1} \ldots P_{n} \cong N_{1}\left(\cong N_{2} \cong \ldots \cong N_{n}\right) .
$$

Proof. This is the restriction to closed terms of a theorem in Barendregt [1984], theorem 14.4.12, having the same proof.

1.13. Corollary. The perpendicular lines lemma is false for any $n>1$, if $\cong$ is replaced by $=_{\beta}$.

Proof (For $n=1$ the perpendicular lines lemma is trivially true for $=_{\beta}$.). Let $n>$ 1. For notational simplicity we assume $n=2$ and give a counter example. Define

$$
\begin{aligned}
& \mathcal{W}_{e_{1}}=\left\{N \in \Lambda^{\varnothing} \mid N=\langle\mathrm{S}, \mathrm{S}\rangle\right\} \\
& \mathcal{W}_{e_{2}}=\left\{N \in \Lambda^{\varnothing} \mid \exists Z \in \Lambda^{\varnothing}[N=\langle\mathrm{I}, Z\rangle \vee N=\langle Z, \mathrm{I}\rangle]\right\}
\end{aligned}
$$

Then $\left\{\mathcal{W}_{e}\right\}_{e \in\left\{e_{1}, e_{2}\right\}}$ is a V-partition. Let $H$ be the combinator obtained from this partition by the main theorem. Then for all $Z \in \Lambda^{\varnothing}$

$$
H\langle\mathrm{~S}, \mathrm{~S}\rangle \neq H\langle\mathrm{I}, Z\rangle=H\langle Z, \mathrm{I}\rangle .
$$

Now define $F \equiv \lambda x y \cdot H\langle x, y\rangle$. Then for all $Z \in \Lambda^{\varnothing}$

$$
F S S \neq F|Z=F Z| \text {. }
$$

This is indeed a counterexample.

We do believe the conjecture in Barendregt [1984], stating that the perpendicular line lemma with $\cong$ replaced by $=_{\beta}$ is correct for open terms. 


\section{Proof of the main theorem}

In order to prove the main theorem 1.5, let a V-partition determined by $S$ be fixed in this section. By proposition 1.4 it may be assumed that the partition is inhabited.

2.1. Lemma. Let $\left\{\mathcal{W}_{e}\right\}_{e \in S}$ be an inhabited V-partition.

(i) There exists a total recursive function $f=f_{S}$ such that

$$
\forall e \in S W_{e}=\left\{f\left((2 e+1) 2^{n}\right) \mid n \in \mathbb{N}\right\} .
$$

(ii) There exists a combinator $\mathrm{E}^{S}$ such that

$$
\forall e \in S \mathcal{W}_{e}=\left\{\mathrm{E}^{S} \mathbf{c}_{(2 e+1) 2^{n}} \mid n \in \mathbb{N}\right\}
$$

Proof. (i) By elementary recursion theory there exists a recursive function $h$ such that $W_{e}=\operatorname{Range}\left(\varphi_{h(e)}\right)$ and $\varphi_{h(e)}$ is total, for all $e \in S$. Observing that $e, n$ are uniquely determined by $k=(2 e+1) 2^{n}$, define $f$ by $f(0)=0, f\left((2 e+1) 2^{n}\right)=$ $\varphi_{h(e)}(n)$.

(ii) Take $\mathrm{E}^{S}=\mathrm{E} \circ F_{S}$, where $F_{S}$ lambda defines $f_{S}$ and $E c_{\# M}=M$ for all $M \in \Lambda^{\varnothing}$.

2.2. Definition. (i) Define

$$
\begin{aligned}
\operatorname{odd}(0) & =0 ; \\
\operatorname{odd}\left((2 e+1) 2^{n}\right) & =2 e+1 .
\end{aligned}
$$

(ii) Define $M \sim N$ iff $M=N \vee M=\mathrm{E}_{m}, N=\mathrm{E}_{n}$ and $\operatorname{odd}(m)=\operatorname{odd}(n)$, for some $m, n$.

Notice that $M \sim N$ iff $M=N$ or $\exists e \in S M, N \in \mathcal{W}_{e}$. Therefore we have to prove that there exists a combinator $H$ such that

$$
H M=H N \Leftrightarrow M \sim N .
$$

The proof consists in constructing a combinator $H=H^{S}$ such that

1. $M \sim N \Rightarrow H M=H N$, proposition 2.4;

2. $H M=H N \Rightarrow M \sim N$, proposition 2.9 .

The second part of the main theorem easily follows by inspecting the proof.

2.3. Definition. (i) Define

$$
\begin{aligned}
T & \equiv \lambda x y z \cdot x y(x y z) \\
A & \equiv \lambda f g x y z \cdot f x(a(\mathrm{E} x))\left[f\left(\mathrm{~S}^{+} x\right) y\left(g\left(\mathrm{~S}^{+} x\right)\right) z\right] \\
B & \equiv \lambda f g x \cdot f(\mathrm{~S} x)\left(a(\mathrm{E}(T x))\left(g\left(\mathrm{~S}^{+} x\right)\right)(g x) .\right.
\end{aligned}
$$

(ii) By the double fixed-point theorem there exists terms $F, G$ such that

$$
\begin{aligned}
& F \rightarrow A F G ; \\
& G \rightarrow B F G .
\end{aligned}
$$


To be explicit, write

$$
\begin{aligned}
D & \equiv(\lambda x y \cdot y(x x y)) \\
Y & \equiv D D ; \\
G & \equiv Y(\lambda u \cdot B(Y(\lambda v \cdot A u v)) u) \\
F & \equiv Y(\lambda u \cdot A u G) .
\end{aligned}
$$

(iii) Finally define

$$
H \equiv \lambda x a . F \mathbf{c}_{1}(a x)\left(G \mathbf{c}_{1}\right) .
$$

Notation. Write

$$
\begin{aligned}
F_{k} & \equiv F \mathbf{c}_{k} ; \\
G_{k} & \equiv G \mathbf{c}_{k} ; \\
\mathrm{E}_{k} & \equiv \mathrm{Ec}_{k} ; \\
a_{k} & \equiv a \mathrm{E}_{k} ; \\
H_{k}[] & \equiv F_{k}[] G_{k} ; \\
C_{k}[] & \equiv F_{k} a_{k}\left([] G_{k}\right) .
\end{aligned}
$$

Note that by construction

$$
\begin{aligned}
F_{k} M N & \rightarrow F_{k} a_{k}\left(F_{k+1} M G_{k+1} N\right) ; \\
G_{k} & \rightarrow F_{k+1} a_{2 k} G_{k+1} G_{k} .
\end{aligned}
$$

By reducing $F$, respectively $G$, it follows that

$$
\begin{aligned}
& H_{k}\left[a_{p}\right] \equiv F_{k} a_{p} G_{k} \rightarrow C_{k}\left[H_{k+1}\left[a_{p}\right]\right] \\
& H_{k}\left[a_{k}\right] \equiv F_{k} a_{k} G_{k} \rightarrow C_{k}\left[H_{k+1}\left[a_{2 k}\right]\right]
\end{aligned}
$$

2.4. Proposition. $M \sim N \Rightarrow H M=H N$.

Proof. By lemma 2.1 it suffices to show $H \mathrm{E}_{k}=H \mathrm{E}_{2 k}$ for all $k$.

$$
\begin{array}{rlrl}
H \mathrm{E}_{k} & =\lambda a \cdot H_{1}\left[a_{k}\right] & \\
& =\lambda a \cdot C_{1}\left[C_{2}\left[\ldots C_{k-1}\left[H_{k}\left[a_{k}\right]\right] . .\right]\right], & & \text { by }(1), \\
& =\lambda a \cdot C_{1}\left[C_{2}\left[\ldots C_{k-1}\left[C_{k}\left[H_{k}\left[a_{2 k}\right]\right]\right] . .\right]\right], & & \text { by }(2), \\
H \mathrm{E}_{2 k} & =\lambda a \cdot H_{1}\left[a_{2 k}\right] & \\
& =\lambda a \cdot C_{1}\left[C_{2}\left[\ldots C_{k-1}\left[C_{k}\left[H_{k}\left[a_{2 k}\right]\right]\right] . .\right]\right], & & \text { by }(1) .
\end{array}
$$


As a piece of art we exhibit in more detail the reduction flow (contracted redexes are underlined).

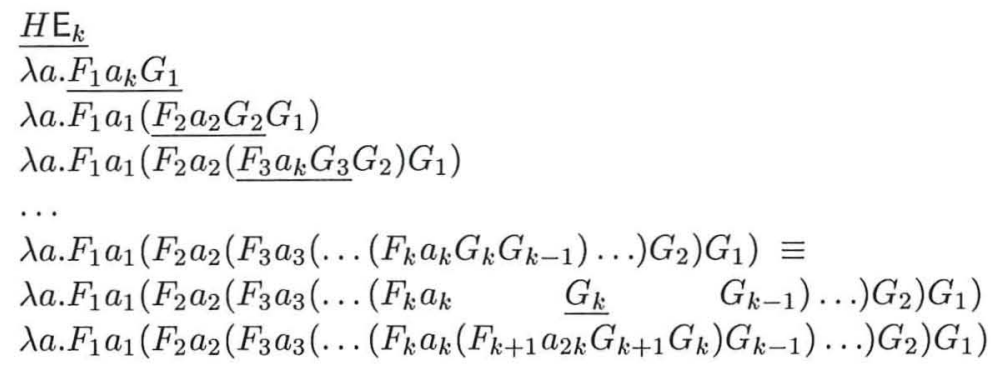

And also

$$
\begin{aligned}
& H \mathrm{E}_{2 k} \rightarrow \ldots \rightarrow \\
& \lambda a . F_{1} a_{1}\left(F_{2} a_{2}\left(F_{3} a_{3}\left(\ldots\left(F_{k} a_{k}\left(F_{k+1} a_{2 k} G_{k+1} G_{k}\right) G_{k-1}\right) \ldots\right) G_{2}\right) G_{1}\right)
\end{aligned}
$$

For the converse implication we need the fine structure of the reduction.

2.5. Definition. Define

$$
\begin{aligned}
D_{k}^{0}[M] & \equiv F_{k}(a M) \equiv Y(\lambda u \cdot A u G) \mathbf{c}_{k}(a M) \\
D_{k}^{1}[M] & \equiv(\lambda y \cdot y(D D y))(\lambda u \cdot A u G) \mathbf{c}_{k}(a M) \\
D_{k}^{2}[M] & \equiv(\lambda u \cdot A u G) F_{k}(a M) \\
D_{k}^{3}[M] & \equiv A F G \mathbf{c}_{k}(a M) \\
D_{k}^{4}[M] & \equiv\left(\lambda g x y z \cdot F_{x}\left(a \mathrm{E}_{x}\right)\left(F_{\mathrm{S}+} y\left(g\left(\mathrm{~S}^{+} x\right)\right) z\right)\right) G \mathbf{c}_{k}(a M) \\
D_{k}^{5}[M] & \equiv\left(\lambda x y z \cdot F_{x}\left(a \mathrm{E}_{x}\right)\left(F_{\mathrm{S}^{+} x} y G_{\mathrm{S}^{+} x} z\right)\right) \mathbf{c}_{k}(a M) \\
D_{k}^{6}[M] & \equiv\left(\lambda y z \cdot F_{k}\left(a \mathrm{E}_{k}\right)\left(F_{\mathrm{S}^{+} \mathrm{c}_{k}} y G_{\mathrm{S}^{+} \mathbf{c}_{k}} z\right)\right)(a M) \\
D_{k}^{7}[M] & \equiv\left(\lambda z \cdot F_{k}\left(a \mathrm{E}_{k}\right)\left(F_{\mathrm{S}^{+} \mathrm{c}_{k}}(a M) G_{\mathrm{S}^{+} \mathrm{c}_{k}} z\right)\right)
\end{aligned}
$$

2.6. Lemma. Let $F_{k}(a M) N$ head-reduce in $8 p+q$ steps to $W$. Then

$$
\begin{aligned}
W & \equiv D_{k}^{q}[M] N, & & \text { if } p=0 ; \\
& \equiv D_{k}^{q}\left[\mathrm{E}_{k}\right]\left(\left(H_{k+1}\left[\mathrm{E}_{k}\right]\right)^{p-1}\left(H_{k+1}[M] N\right)\right), & & \text { else. }
\end{aligned}
$$

Proof. Note that $F_{k}(a M) N \equiv D_{k}^{0}[M] N$. Moreover,

$$
\begin{array}{llll}
D_{k}^{q}[M] N & \rightarrow_{h} & D_{k}^{q+1}[M] N, & \text { for } q<7 ; \\
D_{k}^{7}[M] N & \rightarrow_{h} & D_{k}^{0}\left[\mathrm{E}_{k}\right]\left(H_{k+1}[M] N\right) . &
\end{array}
$$

The rest is clear. At steps 16, 24 we obtain for example

$$
\begin{array}{rll}
D_{k}^{7}\left[\mathrm{E}_{k}\right]\left(H_{k+1}[M] N\right) & \rightarrow_{h} & D_{k}^{0}\left[\mathrm{E}_{k}\right]\left(\left(H_{k+1}\left[\mathrm{E}_{k}\right]\right)\left(H_{k+1}[M] G_{k}\right)\right) . \\
D_{k}^{7}\left[\mathrm{E}_{k}\right]\left(\left(H_{k+1}\left[\mathrm{E}_{k}\right]\right)\left(H_{k+1}[M] G_{k}\right)\right) & \rightarrow_{h} & D_{k}^{0}\left[\mathrm{E}_{k}\right]\left(\left(H_{k+1}\left[\mathrm{E}_{k}\right]\right)^{2}\left(H_{k+1}[M] G_{k}\right)\right) .
\end{array}
$$

Remember that a standard reduction $\sigma: M \rightarrow_{s} N$ always consists of a headreduction followed by an internal reduction:

$$
\sigma: M \rightarrow_{h} W \rightarrow_{i} N .
$$


Notation. Write $M=_{s \leq n} N$ if there are standard reductons of length $\leq n$ from $M$ respectively $N$ to a common reduct $Z$. Similarly $M={ }_{i \leq n} N$ for internal standard reductions. Also the notations $=_{s<n}$ and $=_{i<n}$ will be used.

2.7. LemmA. (i) $D_{k}^{q}[M] N={ }_{i \leq n} D_{k}^{q^{\prime}}\left[M^{\prime}\right] N^{\prime} \Rightarrow q=q^{\prime} \& N={ }_{s \leq n} N^{\prime}$.

(ii) $D_{k}^{q}[M] N={ }_{i \leq n} D_{k}^{q}\left[M^{\prime}\right] N^{\prime} \& q<7 \Rightarrow M={ }_{s \leq n} M^{\prime}$.

(iii) $D_{k}^{7}[M] N={ }_{i \leq n} D_{k}^{7}\left[M^{\prime}\right] N^{\prime} \Rightarrow H_{k+1}[M]={ }_{s \leq n} H_{k+1}\left[M^{\prime}\right]$.

Proof. (i) Suppose $D_{k}^{q}[M] N={ }_{i \leq n} D_{k}^{q^{\prime}}\left[M^{\prime}\right] N^{\prime}$. Then By observing where the free variable $a$ occurs one can conclude that $q=q^{\prime}$. Since the reductions to a common reduct are internal, the positions of $N, N^{\prime}$ are not changed and hence $N={ }_{s \leq n} N^{\prime}$.

(ii) Obvious from the definition of $D_{k}^{q}$.

(iii) In this case it follows that

$$
D_{k}^{0}\left[\mathrm{E}_{k}\right]\left(H_{k+1}[M] z\right)={ }_{i \leq n} D_{k}^{0}\left[\mathrm{E}_{k}\right]\left(H_{k+1}\left[M^{\prime}\right] z\right) .
$$

The conclusion $H_{k+1}[M]={ }_{s \leq n} H_{k+1}\left[M^{\prime}\right]$ depends on the fact that there are the free variables $z$ to mark the residuals.

2.8. Lemma. Suppose $G_{k}={ }_{s \leq n}\left(H_{k+1}\left[\mathrm{E}_{k}\right]\right)^{d}\left(H_{k+1}[M] G_{k}\right)$. Then

$$
H_{k+1}\left[\mathrm{E}\left(T \mathbf{c}_{k}\right)\right]={ }_{s<n} H_{k+1}[M] .
$$

Proof. By induction on $d$. If $d=0$, then we have $G_{k}=_{s \leq n} H_{k+1}[M] G_{k}$. So there are standard reductions of these two terms to a common reduct. Observe that the head-reduction starting with $G_{k}$ begins as follows.

$$
\begin{array}{rl}
G_{k} & \equiv Y(\lambda u \cdot B(Y(\lambda v \cdot A v u)) u) \mathbf{c}_{k} \\
& \rightarrow_{h} \quad(\lambda x \cdot x(Y x))(\lambda u \cdot B(Y(\lambda v \cdot A v u)) u) \mathbf{c}_{k} \\
& \rightarrow_{h} \quad(\lambda u \cdot B(Y(\lambda v \cdot A v u)) u) G \mathbf{c}_{k} \\
& \rightarrow_{h} \quad B F G \mathbf{c}_{k} \\
& \rightarrow_{h} \quad\left(\lambda g x \cdot F\left(\mathrm{~S}^{+} k\right)\left(a\left(\mathrm{E}^{S}(T x)\right)\right)\left(g\left(\mathrm{~S}^{+} k\right)\right)(g x) G \mathbf{c}_{k}\right. \\
\rightarrow_{h} & \left(\lambda x \cdot F\left(\mathrm{~S}^{+} k\right)\left(a\left(\mathrm{E}^{S}(T x)\right)\right)\left(G\left(\mathrm{~S}^{+} k\right)\right)(G x)\right) \mathbf{c}_{k} \\
\rightarrow_{h} & F\left(\mathrm{~S}^{+} k\right)\left(a\left(\mathrm{E}^{S}\left(T \mathbf{c}_{k}\right)\right)\right)\left(G\left(\mathrm{~S}^{+} k\right)\right)\left(G \mathbf{c}_{k}\right) .
\end{array}
$$

The heads of these terms are not of order 0 except the last one. But $H_{k+1}[X]$ is always of order 0 . Therefore the mentioned standard reduction of $G_{k}$ goes at least to this last term $H_{k+1}\left[\mathrm{E}^{S}\left(T \mathbf{c}_{k}\right)\right] G_{k}$. But then $H_{k+1}\left[\mathrm{E}^{S}\left(T \mathbf{c}_{k}\right)\right]={ }_{s<n} H_{k+1}[M]$.

If $d>0$, then start the same argument as above, but at the intermediate conclusion

$$
H_{k+1}\left[\mathrm{E}^{S}\left(T \mathbf{c}_{k}\right)\right] G_{k}==_{s<n}\left(H_{k+1}\left[\mathrm{E}_{k}\right]\right)^{d}\left(H_{k+1}[M] G_{k}\right),
$$

one preceeds by concluding that

$$
G_{k}={ }_{s<n} H_{k+1}\left[\mathrm{E}_{k}\right]^{d-1}\left(H_{k+1}[M] G_{k}\right)
$$

and uses the induction hypothesis.

2.9. Proposition. $H_{k}[M]=H_{k}[N] \Rightarrow M \sim N$. 
Proof. By the standardization theorem it suffices to show for all $n$ that

$$
\forall k \in \mathbb{N}\left[H_{k}[M]={ }_{s \leq n} H_{k}[N] \Rightarrow M \sim N\right] .
$$

This will be done by induction on $n$. From $H_{k}[M]={ }_{s \leq n} H_{k}[N]$ it follows that

$$
\begin{array}{rllll}
H_{k}[M] & \rightarrow_{h} & W_{M} & \rightarrow_{i} & Z \\
H_{k}[N] & \rightarrow_{h} & W_{N} & \gg_{i} & Z .
\end{array}
$$

for some $W_{M}, W_{N}, Z$.

Case 1. $W_{M}, W_{N}$ are both reached after $<8$ steps. Then by lemma $2.6 W_{M} \equiv$ $D_{k}^{q}[M] G_{k}, W_{N} \equiv D_{k}^{q^{\prime}}[N] G_{k}$. By lemma 2.7(i) it follows that $q=q^{\prime}$. If $q<7$, then by 2.7 (ii) one has $M=N$ so $M \sim N$. If $q=7$, then by 2.7 (iii) one has $H_{k+1}[M]={ }_{s<n} H_{k+1}[N]$ and by the induction hypothesis one has $M \sim N$.

Case 2. $W_{M}$ is reached after $p \geq 8$ steps and $W_{N}$ after $q<8$ steps. Then $p=$ $8 d+q$ and, keeping in mind lemma $2.7(\mathrm{i})$, it follows that $W_{M} \equiv D_{k}^{q}[M] G_{k}, W_{N} \equiv$ $D_{k}^{q}\left[E_{k}\right] R, G_{k}={ }_{s<n} R$, where $R \equiv\left(H_{k+1}\left[\mathrm{E}_{k}\right]\right)^{d-1}\left(H_{k+1}[N] G_{k}\right)$. Then as in case 1 it follows that $M \sim \mathrm{E}_{k}$. Moreover, by lemma $2.8 H_{k+1}\left[\mathrm{E}_{2 k}\right]={ }_{s<n} H_{k+1}[N]$, so by the induction hypothesis $\mathrm{E}_{2 k} \sim N$. So $M \sim \mathrm{E}_{k} \sim \mathrm{E}_{2 k} \sim N$.

Case 3 . Both $W_{M}, W_{N}$ are reached after $\geq 8$ steps. Then

$$
\begin{aligned}
W_{M} & \equiv D_{k}^{j}\left[\mathrm{E}_{k}\right]\left(\left(H_{k+1}\left[\mathrm{E}_{k}\right]\right)^{d}\left(H_{k+1}[M] G_{k}\right)\right) ; \\
W_{N} & \equiv D_{k}^{j}\left[\mathrm{E}_{k}\right]\left(\left(H_{k+1}\left[\mathrm{E}_{k}\right]\right)^{d^{\prime}}\left(H_{k+1}[N] G_{k}\right)\right) .
\end{aligned}
$$

If $d=d^{\prime}$, then by lemma 2.7

$$
\left(H_{k+1}\left[\mathrm{E}_{k}\right]\right)^{d}\left(H_{k+1}[M] G_{k}\right)=_{s<n}\left(H_{k+1}\left[\mathrm{E}_{k}\right]\right)^{d}\left(H_{k+1}[N] G_{k}\right),
$$

so

$$
H_{k+1}[M]={ }_{s<n} H_{k+1}[N],
$$

since $H_{k+1}[X]$ is always of order 0 . Therefore by the induction hypothesis $M \sim N$.

If on the other hand, say, $d<d^{\prime}$, then (writing $d^{\prime}=d+e$ )

$$
\begin{aligned}
& W_{M} \equiv D_{k}^{j}\left[\mathrm{E}_{k}\right]\left(\left(H_{k+1}\left[\mathrm{E}_{k}\right]\right)^{d}\left(H_{k+1}[M] \quad G_{k}\right)\right) ; \\
& W_{N} \equiv D_{k}^{j}\left[\mathrm{E}_{k}\right]\left(\left(H_{k+1}\left[\mathrm{E}_{k}\right]\right)^{d}\left(H_{k+1}\left[\mathrm{E}_{k}\right]\left(\left(H_{k+1}\left[\mathrm{E}_{k}\right]\right)^{e-1}\left(H_{k+1}[N] G_{k}\right)\right)\right)\right) \text {. }
\end{aligned}
$$

so

$$
\begin{array}{rll}
H_{k+1}[M] & ={ }_{s<n} & H_{k+1}\left[\mathrm{E}_{k}\right] \\
G_{k} & ={ }_{s<n} & \left(H_{k+1}\left[\mathrm{E}_{k}\right]\right)^{e-1}\left(H_{k+1}[N] G_{k}\right),
\end{array}
$$

since $H_{k+1}[X]$ is always of order 0 . Therefore by lemma 2.8

$$
H_{k+1}\left[\mathrm{E}_{2 k}\right] \quad{ }_{s<n} \quad H_{k+1}[N]
$$

Therefore by the induction hypothesis twice we obtain $M \sim \mathrm{E}_{k} \sim \mathrm{E}_{2 k} \sim N$.

\section{References}

Barendregt, H. P. [1984]. The Lambda Calculus: its syntax and semantics, revised edition, North-Holland Publishing Co., Amsterdam. 
Rogers, Hartley, Jr. [1987]. Theory of recursive functions and effective computability, second edition, MIT Press, Cambridge, Mass.

Statman, Rick [1986]. Every countable poset is embeddable in the poset of unsolvable terms, Theoret. Comput. Sci. 48(1), pp. 95-100.

Statman, Rick [1998]. Morphisms and partitions of V-sets, CSL'98, Springer, Berlin. To appear.

Statman, Rick [1999]. Consequences of a theorem of Jacopini: consistent equalities and equations, TLCA'99, Springer, Berlin. To appear.

Visser, Albert [1980]. Numerations, $\lambda$-calculus \& arithmetic, To H. B. Curry: essays on combinatory logic, lambda calculus and formalism, Academic Press, London, pp. 259284. 
